\title{
A DEFLECTION POTENTIOMETER FOR VOLTMETER TESTING.
}

By H. B. Brooks.

\section{INTRODUCTION.}

In a former paper $^{1}$ the writer derived an expression for the current through the galvanometer of an unbalanced potentiometer, used in connection with a volt box, and showed how this formula could be applied to the design of a class of instruments, for current and voltage measurements, having properties intermediate between those of the balance type and of the deflection type. A description was given of an instrument constructed on this principle, which was used for measuring voltage in photometric work, and which has now been in service for the past two years, giving satisfactory speed and accuracy.

The object of the present paper is to give some further modifications of circuits which may be used for this purpose, with their advantages and defects; to show what features should be incorporated in an instrument of this kind for such work as the testing of voltmeters, and to describe an instrument of this type recently constructed. This has been designed for voltmeter testing and other precision measurements of voltage in the laboratory and testing room.

\section{A STANDARD INSTRUMENT FOR VOLTMETER TESTING.}

An instrument to be used as a standard for voltmeter testing should first of all be accurate, permanent, and unaffected by magnetic fields and similar disturbing influences. This requires that the bulk of the unknown voltage be measured by the potentiometer method, as no known type of voltmeter, not even so-called "laboratory standard" instruments, will meet the above requirements. In order

'This Bulletin, 2, p. 225; I906. 
to save time and avoid the necessity for extreme steadiness of the test voltage, a small portion of the quantity should be measured by the deflection of a galvanometer. This galvanometer should be a pivoted instrument, of good working qualities, and should have a sensibility such that one division of its scale corresponds to say onetenth to one-fifth of a scale division of a voltmeter of the same maximum range as the potentiometer. This gives great ease of reading, as it is usually sufficient to read the galvanometer to the nearest division, neglecting tenths. These requirements indicate a deflection potentiometer, and as a number of variations are possible, in the arrangement of circuits for such an instrument, these various plans will be examined with a view of determining the one which is the most suitable.

It is desirable that the standard instrument be semiportable, for while such an instrument is commonly used in one place, it is often necessary to make precision measurements in other places on relaatively short notice. In the case of the deflection potentiometer, by building the pivoted galvanometer in as a part of the apparatus, the only accessories are the storage battery and the standard cell; the former must be carried separately, and preferably the latter also.

\section{ARRANGEMENTS OF CIRCUITS.}

In plan I (see page 23I, former article) the volt box ratio is constant, and the setting is made on the potentiometer wire. The arrangement of circuits may be seen from Fig. 4 of this article by omitting the resistance $r_{6}$; the symbols used correspond with those of the previous article. The difficulty with this plan lay in the fact that the compensating resistance in the galvanometer circuit, $r_{4}$, was a function of two variables, the resistance of the potentiometer wire up to the setting, $r_{1}$, and the regulating rheostat in the storage cell circuit, $r_{3}$, no satisfactory way of providing this double compensation being available. To avoid this difficulty plan 2 was devised, in which $r_{1}$ is a constant; $r_{4}$ therefore compensates only for changes in $\Sigma r$ (the total resistance in the galvanometer circuit) due to variations of $r_{3}$. The setting is then made on the volt box, and a compensating resistance $r_{5}$ is arranged to take care of variations due to changes of the setting. This plan was adopted for the first instrument of this type, in which the range was from 95 to r 25 volts. 
A third plan, suggested by Dr. M. G. Lloyd, is like plan 2, except that instead of a constant total resistance $R$ between the emf. terminals of the volt box, with the drop taken from a variable fraction $\frac{\mathrm{I}}{p}$ of this, the drop is taken from a fixed resistance and $R$ is variable, as shown in Fig. I.

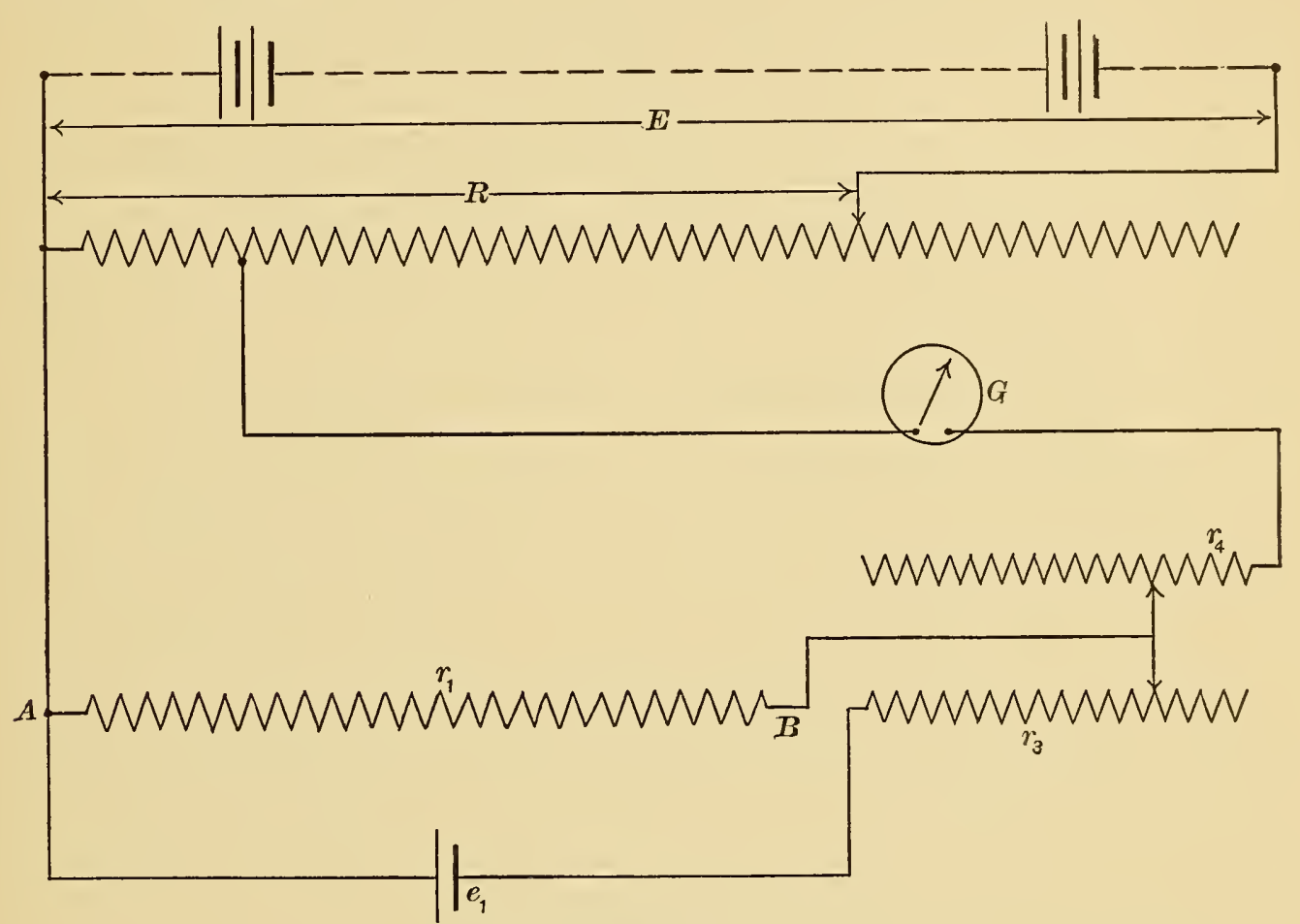

Fig. 1.-Third plan of circuits.

The equations for this case are the same as for plan 2. $R \frac{p-I}{p^{2}}$ is more nearly constant in plan 3 than in plan 2. A serious disadvantage of plan 3 is the fact that $R$, the resistance of the volt box, is low on low settings. If the instrument reads down to a small fraction of the maximum, and through mistake the maximum pressure is applied while the dial is set at or near the minimum, a current many times greater than normal will flow through the part of the volt box in circuit, with consequent damage.

Plans 2 and 3 have the disadvantage of variable damping of the galvanometer. For an instrument of limited range (as 95 to 125 volts) this disadvantage practically disappears. By designing the coils so that the value of $\Sigma r$ for the mean setting is equal to the total resistance for critical damping of the galvanometer, the damp- 
ing will still be such as to give good working, even at the extremes of the range. If, however, we are to cover a wide range with an instrument using plan 2, it is necessary to modify the circuits so as to preserve critical danping over the whole range. This may be accomplished by the arrangement shown in Fig. 2.

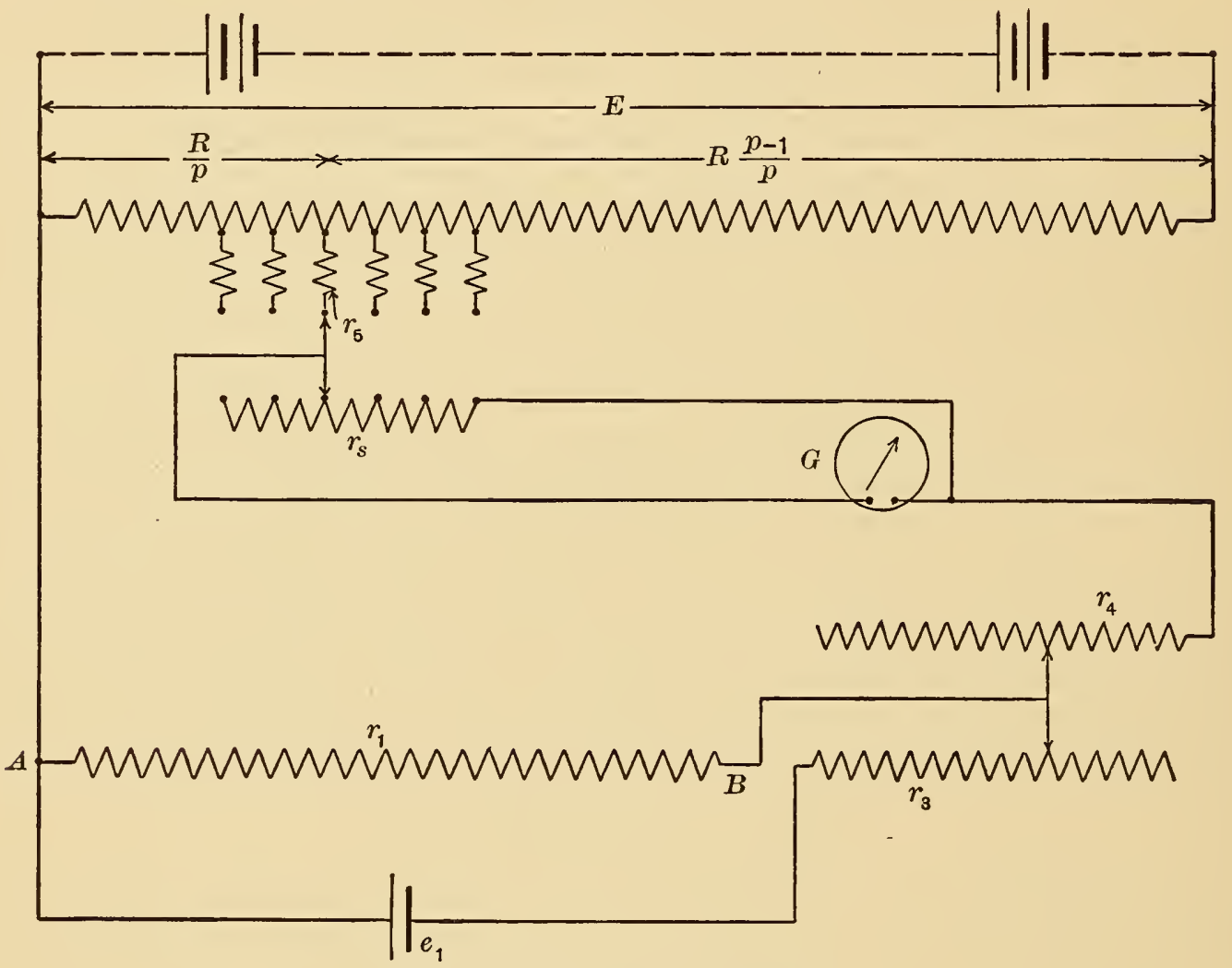

Fig. 2.-Fourth plan of circuits.

This arrangement is the same as plan 2, with the addition of a variable shunt $r_{s}$ to the galvanometer, which is changed at the same time as the compensating resistance $r_{5}$. Since $r_{5}$ and $r_{s}$ are independent variables, we may impose two conditions: (I) The current through the galvanometer, for all settings, must be such that one volt $\Delta E$ (difference between $p$ times the setting and the applied voltage $E$ ) will give a deflection of $m$ scale divisions; (2) the resistance of the damping circuit must be constant and equal to $r_{a}$, the total resistance for aperiodic damping. In the former article it was shown that if $\Sigma r$ is the total resistance in the galvanometer circuit, (neglecting all electromotive forces) $\frac{\mathrm{I}}{p}$ the fractional part of the volt box from which the drop is taken, $m$ the number of scale 
divisions which the galvanometer is to give for one volt unbalanced emf. in the pressure to be measured, the value of $\Sigma r$ is given by the equation

$$
\Sigma r=\frac{\mathrm{I}}{p m I}
$$

If the current $I$ is required by the unshunted galvanometer for one scale division, when the shunt $r_{s}$ is applied this is increased to

$$
\frac{r_{s}+r_{g}}{r_{s}} I
$$

By equation (II) we may write for this case

$$
\Sigma r=\frac{r_{s}}{p m I\left(r_{s}+r_{g}\right)}
$$

For convenience we may denote the resistance in the galvanometer circuit, aside from $r_{s}$ and $r_{g}$, by $r^{\prime}$; or

$$
r^{\prime}=r_{4}+\frac{r_{1} r_{3}}{r_{1}+r_{3}}+R \frac{p-\mathrm{I}}{p^{2}}+r_{5}
$$

The damping circuit is then as shown in Fig. 3, and for critical damping

$$
\begin{aligned}
& r_{g}+\frac{r_{s} r^{\prime}}{r_{s}+r^{\prime}}=r_{a} \\
& \Sigma r=r^{\prime}+\frac{r_{s} r_{g}}{r_{s}+r_{g}}
\end{aligned}
$$

By solving these equations we get

$$
\begin{aligned}
& r_{s}=\frac{r_{a}-r_{g}}{\mathrm{I}-p m I r_{a}} \\
& r^{\prime}=\frac{r_{a}-r_{g}}{p m I r_{a}}
\end{aligned}
$$

Since

$$
r^{\prime}=r_{4}+\frac{r_{1} r_{3}}{r_{1}+r_{3}}+R \frac{p-I}{p^{2}}+r_{5}
$$




$$
\begin{aligned}
\frac{r_{a}-r_{g}}{p m I r_{a}} & =\text { constant }+R \frac{p-\mathrm{I}}{p^{2}}+r_{5} \\
r_{5} & =\frac{r_{a}-r_{g}}{p m I r_{a}}-R \frac{p-\mathrm{I}}{p^{2}}-\text { constant. }
\end{aligned}
$$

By means of these formulæ we may determine the values of $r_{s}$ and $r_{5}$ for a given case. ${ }^{2}$

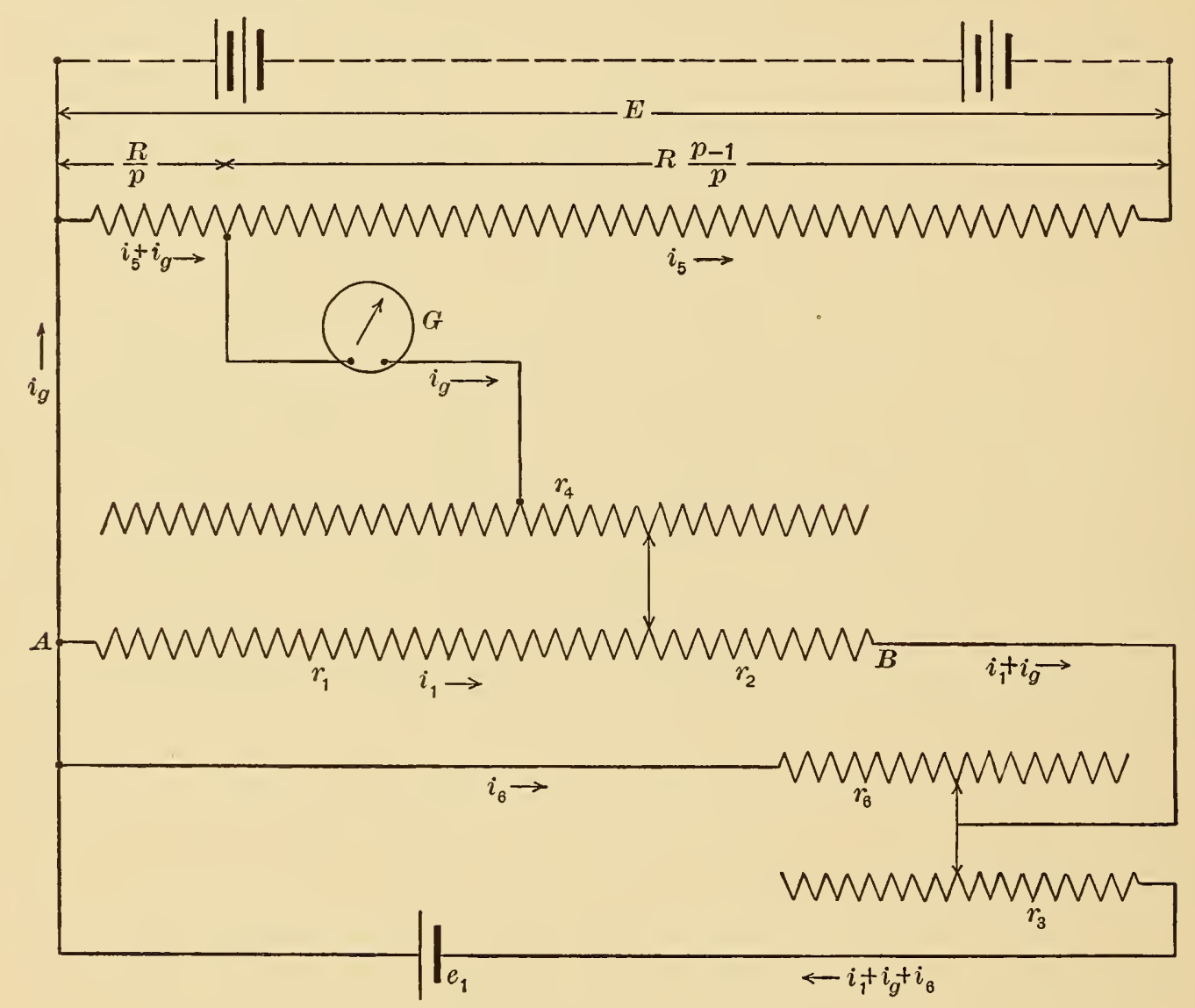

Fig. 4.-Fifth plan of circuits.

The variable shunt to the galvanometer in plan 4 must be made of copper, and should preferably be mounted inside the galvanometer case, to insure equality of temperature. This would require numerous leads from the shunt to the potentiometer, if the galvanometer is not to be built into the potentiometer. Plan 4 also has the disadvantage of requiring a larger number of coils than the

${ }^{2}$ The method of arranging the compensating coils $r_{5}$ in Fig. 2 was suggested to the writer by Dr. W. P. White, who has recently described it in the Physical Review, 95, 334, I907; and in the Zeitschrift für Instrumentenkunde, 27, 210; I907. 
others for a given range and number of steps. A further practical disadvantage of plans 2 and 4 is that the sections of the voltbox, which require precision adjustment, will nearly all be odd values.

In considering the features which would be desirable in an instrument of this class for voltmeter testing, in which the range from zero to the maximum is to be covered, and several ranges provided, it was found that none of these plans entirely met the requirements, and a plan was sought which would be satisfactory. This was obtained from plan I by adding a variable shunt to the potentiometer wire $\mathrm{AB}$, which is varied at the same time as $r_{3}$. This plan is shown in Fig. 4.

In the former article it was shown that for plan $I$, in which the resistance $r_{6}$ is absent, the current through the galvanometer is given by the equation

$$
i_{g}=\frac{\left(\frac{r_{1}}{r_{1}+r_{2}+r_{3}}\right) e_{1}-\frac{E}{p}}{r_{4}+r_{g}+\frac{r_{1}\left(r_{2}+r_{3}\right)}{r_{1}+\left(r_{2}+r_{3}\right)}+R \frac{p-\mathrm{I}}{p^{2}}}
$$

In plan 5 , as $r_{3}$ is increased, $r_{6}$ is decreased; both of these changes tend to lower the fall of potential on AB. By applying Kirchhoff's laws to this network we get for the value of the galvanometer current

$$
i_{g}=\frac{\left(\frac{r_{1}}{r_{1}+r_{2}+\frac{r_{1}+r_{2}+r_{6}}{r_{6}} r_{3}}\right) e_{1}-\frac{E}{p}}{r_{4}+r_{g}+\frac{r_{1}\left(r_{2}+\frac{r_{3} r_{6}}{r_{3}+r_{6}}\right)}{r_{1}+\left(r_{2}+\frac{r_{3} r_{6}}{r_{3}+r_{6}}\right)}+R \frac{p-\mathrm{I}}{p_{2}}}
$$

By comparing this with equation (6) we see that the effect of $r_{6}$ in the first term of the numerator is to make the coefficient of $r_{3}$ greater than unity; that is, $r_{6}$ assists $r_{3}$ in lowering the fall of potential on $\mathrm{AB}$. The third term in the denominator is seen to be $r_{1}$ in parallel with the sum of $r_{2}$ and the resultant of $r_{3}$ and $r_{6}$ in parallel. If, therefore, we can choose corresponding values of $r_{3}$ and $r_{6}$ so as to keep constant the resultant of these two in parallel, while giving the desired regulation of the current through $A B$, 
this third term will be a function of $r_{1}$, since $r_{1}+r_{2}$ is constant $r_{4}$ may then be readily arranged to compensate for this variation, keeping the denominator, $\Sigma r$, the total resistance in the galvanometer circuit, a constant for all settings and all positions of the regulating rheostat $r_{3}$. It should be noted that if we make $r_{6}=\infty$ in equation ( 7 ), it becomes identical with equation (6).

The practical limitations of this method should be examined, for while negative values of resistance may satisfy an equation, such resistances cannot be realized. First, $r_{3}$ cannot be reduced to zero, for this would make the term $\frac{r_{3} r_{6}}{r_{3}+r_{6}}$ always equal to zero, and no regulation would be possible. It is therefore necessary to have a value of $e_{1}$, when the cells are giving the lowest emf. at which it is desired to use them, which is somewhat greater than the fall of potential to be maintained on AB. Let this fall of potential be represented by $a$; then from a consideration of the first term of the numerator in equation ( $\mathrm{I} 7$ ) we have

$$
\begin{gathered}
r_{1}+r_{2}+\frac{r_{1}+r_{2}+r_{6}}{r_{6}} r_{3}=\frac{e_{1}}{a}\left(r_{1}+r_{2}\right) \\
\frac{r_{1}+r_{2}+r_{6}}{r_{6}} r_{3}=\frac{e_{1}-a}{a}\left(r_{1}+r_{2}\right) \\
\frac{r_{w}+r_{6}}{r_{6}} r_{3}=\frac{e_{1}-a}{a} r_{w}
\end{gathered}
$$

where $r_{w}$ is put for $r_{1}+r_{2}$, the resistance of the wire AB. On account of the change of emf. of the storage cell, $e_{1}$ will vary between certain limits, and values of $r_{3}$ and $r_{6}$ must be found for a series of values of $e_{1}$. It is first necessary to find, from equation (II), the constants of the galvanometer and assigned or determined values of $p, m$, and $R$, how large a value we may allow for the maximum resistance between the point of contact on the wire $A B$ and the point A. Some resistance should be reserved in a calibrating coil in the galvanometer circuit, which may be altered, if necessary, to correct for change in the galvanometer sensibility with time. This maximum value, $r_{m}$, will occur when the slider is at the center of the total resistance in the storage cell circuit; that is, when 


$$
r_{1}=\frac{r_{w}}{2}+\frac{r_{3} r_{6}}{2\left(r_{3}+r_{6}\right)}
$$

and its value is

$$
r_{m}=-\frac{r_{w}}{4}+\frac{r_{3} r_{6}}{4\left(r_{3}+r_{6}\right)}
$$

By solving (I8) for $r_{3}$ and substituting this value in (I9), solving for $r_{6}$, we get

$$
r_{6}=\frac{e_{1}}{a} \cdot \frac{4 r_{m}-r_{w}}{\frac{e_{1}}{a}-\frac{4 r_{m}}{r_{w}}}
$$

Substituting this value of $r_{6}$ in equation (I8) and solving for $r_{3}$, we get

$$
r_{3}=\frac{e_{1}}{a} \cdot \frac{r_{w}\left(4 r_{m}-r_{w}\right)}{4 r_{m}}
$$

In this equation $e_{1}$ is the only variable, hence

$$
r_{3}=\text { constant } \times e_{1}
$$

which is a very convenient formula to use.

It is somewhat more convenient to get $r_{6}$, not by using equation (20), but by putting it into the form

$$
\begin{aligned}
r_{6} & =\frac{\left(4 r_{m}-r_{w}\right) r_{3}}{r_{3}-\left(4 r_{m}-r_{w}\right)} \\
& =\frac{k r_{3}}{r_{3}-k}
\end{aligned}
$$

and using this after values of $r_{3}$ have been determined for the various steps of the rheostat.

Examining equation (20), we see that $r_{6}$ will be infinite when

$$
\frac{e_{1}}{a}=\frac{4 r_{m}}{r_{w}}
$$

except when $\frac{e_{1}}{a}$ is also equal to unity, in which case $r_{6}=\frac{0}{0}$. This is not a practical case. When $\frac{e_{1}}{a}$ is less than $\frac{4 r_{m}}{r_{w}}, r_{6}$ will be negative. 
In a practical case this can not occur; $r_{m}$ must be greater than $\frac{r_{w}}{4}$, to allow for regulation, and hence the numerators of the expressions for $r_{3}$ and $r_{6}$ will always be positive. As $r_{m}$ is determined by other considerations, we may choose $r_{w}$ so as to give suitable values for $r_{6}$. Since $r_{\mathrm{w}}$ must be less than $4 r_{m}$ and greater than $\frac{\alpha}{e_{1}} \times 4 r_{m}, \frac{a}{e_{1}}$ being somewhat less than unity, we may give $r_{w}$ the mean of the two possible values, or

$$
\begin{aligned}
r_{w} & =\frac{\mathrm{I}}{2}\left(\mathrm{I}+\frac{a}{e_{1}}\right) 4 r_{m} \\
& =\frac{2\left(a+e_{1}\right) r_{m}}{e_{1}}
\end{aligned}
$$

The value of $e_{1}$ used in this formula should be the lowest emf. at which the storage battery is to be used. It is seen that the nearer $e_{1}$ approaches $a$, or the less margin we allow for regulation, the narrower are the limits for $r_{w}$. It is desirable to have each step of $r_{w}$ an even value, as IO, 20,50, or IoO ohms, so that if the above formula gives a value for each step, which is near an even value, the even value should be used, making the necessary adjustment of values elsewhere.

In the case of plan 5, the fine adjustment rheostat in the storage battery circuit calls for more attention than would usually be necessary. The steps on the series rheostat in the battery circuit being equal in resistance, it would ordinarily be sufficient to give the fine rheostat a resistance equal to or slightly greater than the resistance of one step of the main rheostat. In the case of plan 5, however, this would not be sufficient. When the coarse rheostat is changed one step, both $r_{3}$ and $r_{6}$ are changed; when the fine rheostat is changed, $r_{3}$ only is changed. It is not practicable to use a double fine rheostat, such that $r_{6}$ as well as $r_{3}$ will be changed, on account of the large variation in the value of a step in $r_{6}$, for uniform steps in $r_{3}$. In the instrument about to be described the change in the shunt resistance $r_{6}$ is more effective in changing the current through the potentiometer wire than the change of $r_{3}$, and to produce, by a variation of $r_{3}$ alone, an effect equal to that due to changing $r_{3}$ and $r_{6}$, requires a relatively large change in $r_{3}$. 
Let a fine adjustment rheostat $r_{8}$ be connected so as to form a continuation of $r_{3}$ (Fig. 4). The current through the wire AB, when no current flows through the galvanometer, will be, by equation (I7),

$$
i=\frac{e_{1}}{r_{w}+\frac{r_{w}+r_{6}}{r_{6}} r_{3}}
$$

If we reduce $r_{3}$ by one step, $\Delta r_{3}$, increasing $r_{6}$ by $\Delta r_{6}$, the current will increase. The insertion of the fine rheostat $r_{8}$ in series with $r_{3}$ should reduce the current to the original value; hence,

$$
r_{w}+\frac{r_{w}+r_{6}}{r_{6}} r_{3}=r_{w}+\frac{r_{w}+r_{6}+\Delta r_{6}}{r_{6}+\Delta r_{6}}\left(r_{3}-\Delta r_{3}+r_{8}\right)
$$

from which

$$
r_{8}=\Delta r_{3}+\frac{r_{3} r_{w} \Delta r_{6}}{r_{6}\left(r_{w}+r_{6}+\Delta r_{6}\right)}
$$

This shows that $r_{8}$ must always be greater than $\Delta r_{3}$, unless $r_{6}$ be made infinite, which gives plan $I$. The expression also shows that the amount by which $r_{8}$ is in excess of $\Delta r_{3}$ will increase with increasing $r_{3}, r_{w}$, and $\Delta r_{6}$, and decrease with increasing $r_{6}$. It will be found that the value of $r_{8}$ for a given instrument will not be the same at all points of the coarse rheostat. In the present instrument, $r_{8}$ should be 7.5 ohms when the coarse rheostat is in the position of maximum $r_{3}$, and I0.4 ohms for minimum $r_{3}$. It is desirable to have a small amount of overlap, hence this rheostat was made of II ohms resistance.

It is often desirable to use an external resistance or "multiplier" to increase the range of a voltmeter. The same thing can be done with the deflection potentiometer, though not with the same facility for every plan. With plans I and 5, an external inultiplier can be used to increase the range, with a small (usually negligible) error which is a constant per cent of the deflection reading, for any setting of the main dial. This may also be done with plan 2, if the range is not great; but the relative error in the deflection then varies with the setting, being inversely proportional to $p$. It is impracticable to use a multiplier with plan 3 , as the resistance of the multiplier would have to change with the setting, so as to be always the 
same multiple of the variable $R$. The general expression for the error involved in the use of an external multiplier is given in a later paragraph.

It will be seen that plan 5 is the most suitable one for a standard instrument for voltmeter testing, having the most practical points of advantage in construction and operation. The volt box resistance is high and constant; the resistance of the galvanometer circuit is constant, and the galvanometer may thus be critically damped at all settings, without the use of shunts. The steps on the main dial are

4. DESCRIPTION OF INSTRUMENT.

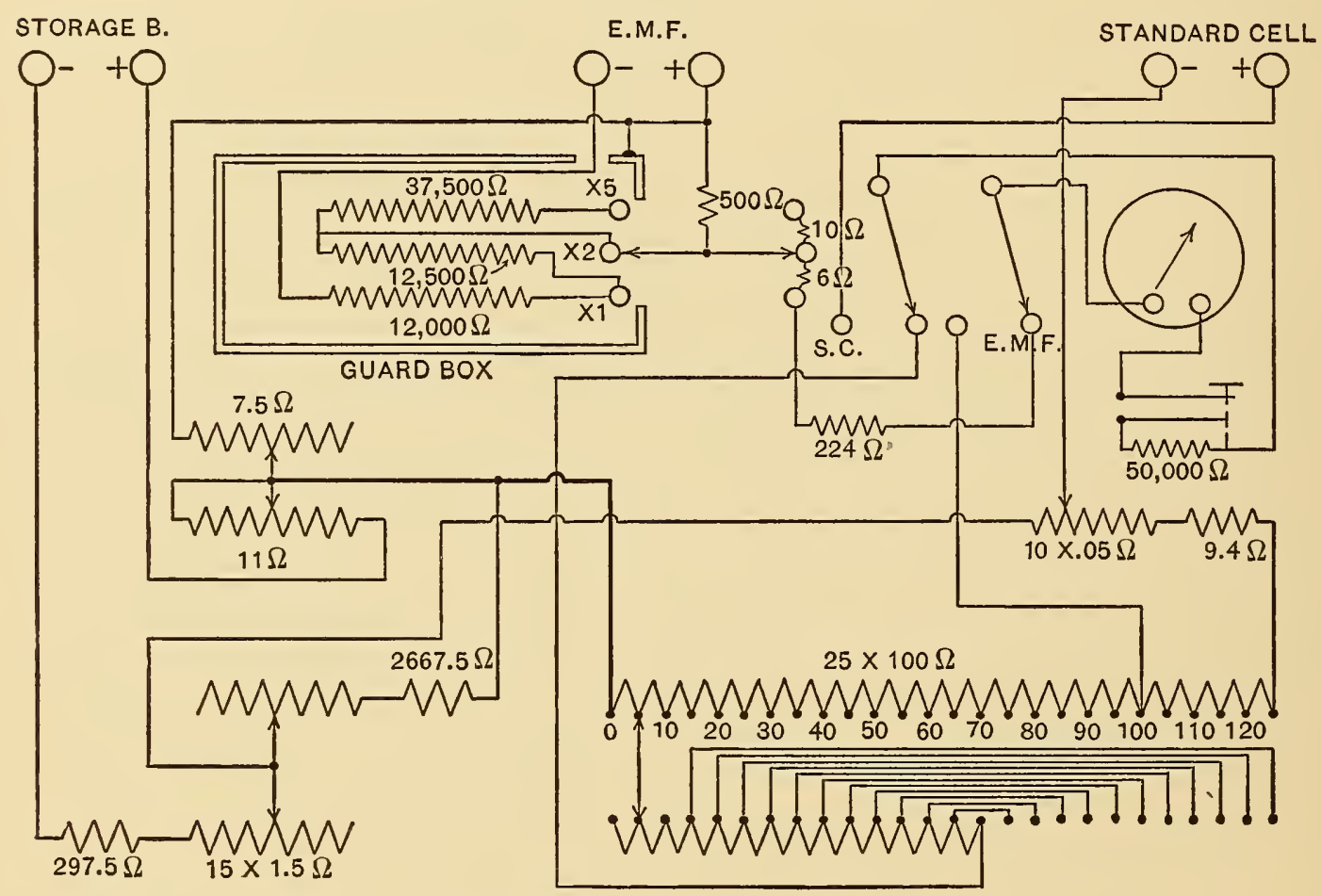

Fig. 5.-Connections of potentiometer (diagrammatic).

equal resistances, and by suitable design may also be made even values, which facilitates checking and adjusting. Multiple ranges are readily provided, and if it is desired to measure pressures in excess of the highest for which the instrument is built, this may be done by the use of an external multiplier, giving a very small but constant percentage correction to the deflection reading.

An instrument has been designed in accordance with plan 5, for use in voltmeter testing in the Bureau of Standards. A diagrammatic plan of connections is given in Fig. 5 . 
The main dial has 25 steps of roo ohms each. This is in series with a coil of $9.4 \mathrm{ohms}$ and a dial of Io steps of .05 ohm each. The Weston standard cell is balanced around 509.4 ohms plus the amount on the dial, and as the standard current is .002 ampere, cells of 1.0188 to I.org 8 volts may be used, and if need be, this range may be varied by changing the $9.4 \mathrm{ohm}$ coil. In the storage cell circuit is a series rheostat whose minimum resistance is $297.5 \mathrm{ohms}$, increasing from this value in I 5 steps of $I .5$ ohms each. This is $r_{3}$ of the preceding discussion. At the same time that $r_{3}$ is increased, the resistance in shunt to the potentiometer wire, $r_{6}$, decreases from a maximum of $6,8 \mathrm{r} 4$ ohms to a minimum of $2,667.5$ ohms. A fine rheostat of II ohms in the battery circuit covers any step of the coarse rheostat, and has a compensating resistance of $7.5 \mathrm{ohms}$ maximum in the galvanometer circuit.

The drop is taken from the ends of a 500 ohm coil, which is in series with $\mathrm{I} 2,000 \mathrm{ohms}$ when the range switch is set on $\times \mathrm{r}$. When this switch is at $\times 2$ and $\times 5$ the total resistance between the emf. posts is 25,000 and 62,500 ohms, respectively. All of these coils, except the first $500 \mathrm{ohms}$, are mounted within and well insulated from a brass box which entirely encloses them. The negative emf. terminal post is inside of, and well insulated from, a brass sleeve which projects into the box and is soldered to it. This box is connected by a wire to the positive emf. post, and acts as a "guard wire" to prevent leakage currents from flowing through the circuits of the potentiometer proper. This is a very important precaution, which will be appreciated when we consider the high pressure $(625$ volts) available for producing leakage, and the fact that the full deflection of the galvanometer is produced by .00006 ampere, a current which 625 volts would send through a resistance of over ten megohms; while a current sufficient to give a readable deflection would flow, under this pressure, through 3,00o megohms. With the arrangement shown, the maximum pressure which may produce leakage through the galvanometer is 6 volts.

The main dial has a set of compensating coils, $r_{4}$, for keeping constant the resistance between the sliding contact and the o end of this dial. By arranging the values of $r_{3}, r_{6}$, and the standard cell coils so that the resultant resistance beyond the 125 end of the dial is 300 ohms, the total resistance in the storage battery circuit being

1 $1737-07-8$ 
2,800 ohms, the point of maximum resultant resistance to the galvanometer current will come at the setting 70 , when $r_{1}$ is 1,400 ohms; the resistance at 75 will be the same as at 65 , and so on. Hence compensating coils are used up to the point 70 , and by the use of cross connections no additional coils are needed beyond this point.

A double-pole double-throw switch is used to change from standard cell to emf., and a push button is provided in the galvanometer circuit. This button operates a double successive contact key having a protective resistance of $50,000 \mathrm{ohms}$ in the first contact. The fine adjustment rheostat consists of two semicircular coils of insulated wire, the insulation being removed on the face to allow the sliding brush to make contact.

While the lowest range provided is nominally o to I 25 volts, the range o to 5 volts may be had by using as the - emf. terminal the lever of the range switch, and adding sufficient resistance to the galvanometer circuit to make the proper total. In other words, the normal range of the potentiometer is from o to 5 volts, readable to .0004 volt (one-tenth of a scale division of the galvanometer). This range may be increased as desired by adding resistance in the emf. circuit at the rate of 100 ohms per volt. The reason for not making the lower ranges immediately available by setting the range switch is the danger of accident to these low ranges, due to the inadvertent application of the usual working pressures of roo volts and over. The lower ranges are not frequently required, and may be arranged for specially as above described. It is proposed to construct an external rheostat which may be quickly connected up to give the ranges o to I 2.5 , O to 25 , and o to 62.5 volts, the proper compensating resistances being thrown into the galvanometer circuit for each range. The presence of this special accessory apparatus would constitute an indication of the need for caution as to the value of the voltage to be applied.

A view of the instrument is shown in Fig. 6, from which it will be seen that the galvanometer is built into the rubber top, making the apparatus self-contained with the exception of the cells. The scale of the galvanometer is shown in Fig. 7. It has two sets of figures; an upper with $O$ in the center, and a lower with 5 in the center. Ten divisions of this scale correspond to one volt, the 


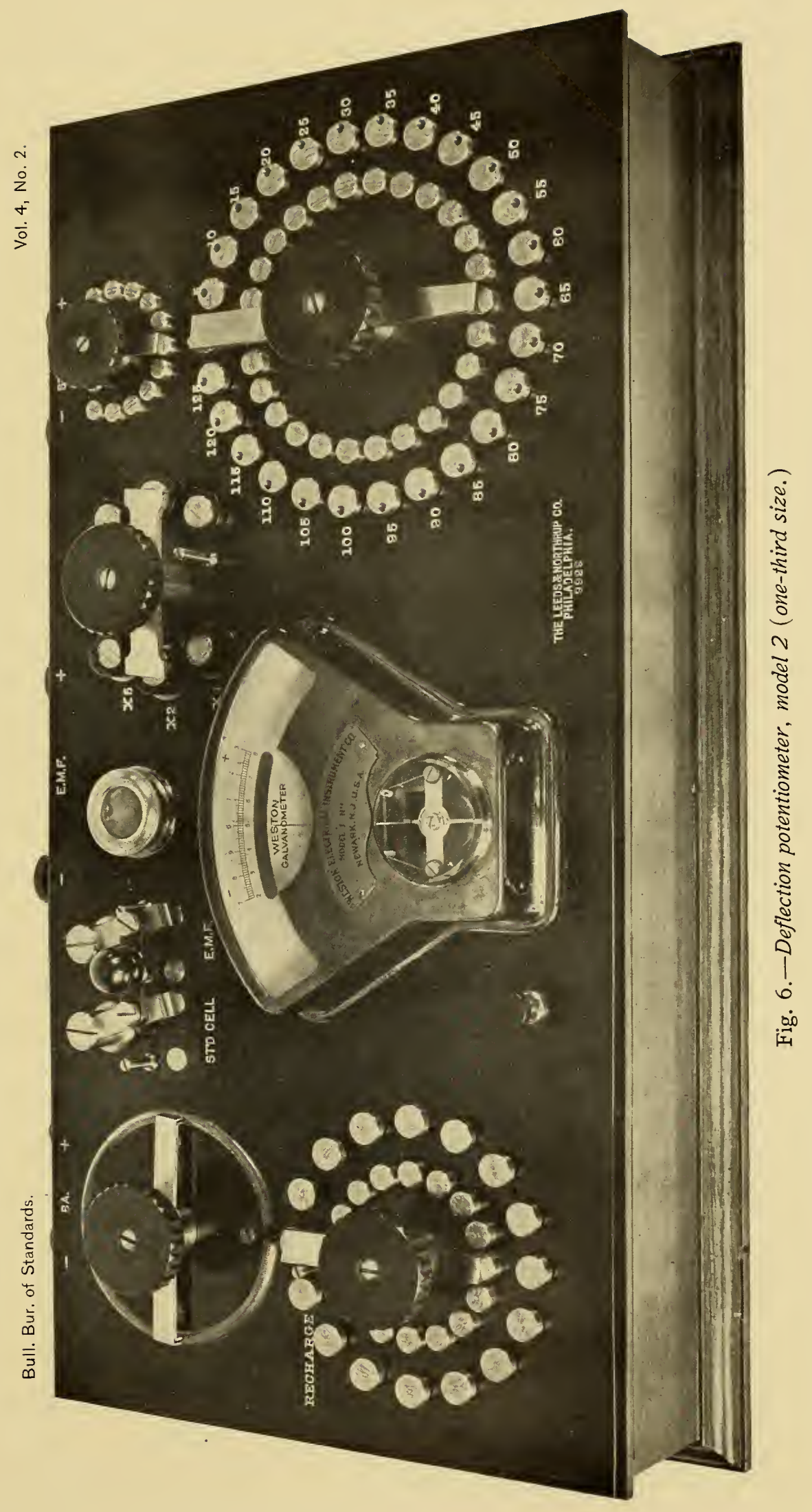


upper set of figures being used when the dial reading ends in 0 , the lower set when it ends in 5. Thus, if the dial reads IIO and the galvanometer needle stands at 22 divisions to the right, the emf. to be measured is IIO $+2.2=\operatorname{II} 2.2$ volts. In practice, the dial reading is mentally set at the center of the galvanometer scale, using upper or lower figures as required, when the value of the voltage can be read off from the scale, reading directly to tenths of a volt and estimating hundredths. The above applies to the first or principal range; when the range switch is at $\times 2$ or $\times 5$ the operation of the instrument is exactly the same, but the values are to be multiplied by 2 or 5 , giving a maximum range of 625 volts. Should it be necessary to extend this by using a multi-

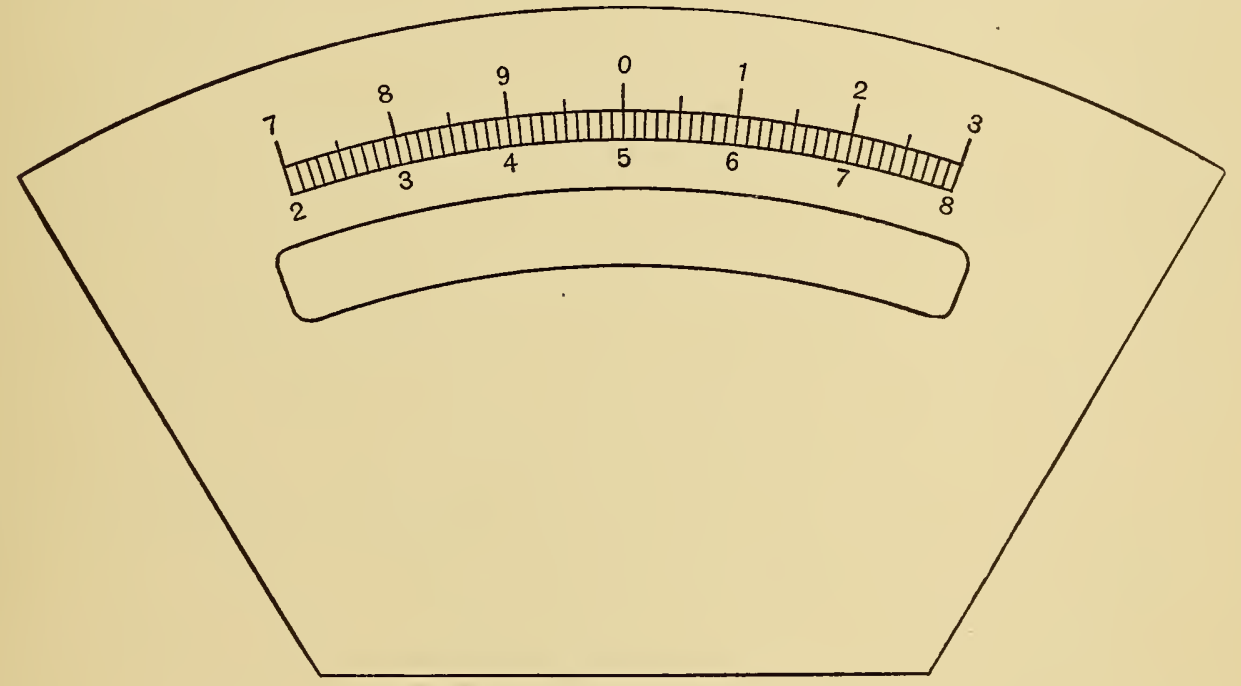

Fig. 7.-Galvanometer scale.

plier, this may be done with an error less than 0.2 per cent of the deflection portion of the reading. At full scale deflection this is about .05 division, or about the limit of setting the needle on a line. Hence, in this design, multipliers introduce a negligible error.

This instrument was built by The Leeds \& Northrup Company, of Philadelphia, $\mathrm{Pa}$, the galvanometer being supplied by the Weston Electrical Instrument Company, of Newark, N. J. It is so rapid and dead-beat in operation that the only limit to the rapidity of testing voltmeters with it is the ability of the operator to control the voltage and read the voltmeter under test. The appliances now in use at the Bureau for voltage control will be replaced soon by others which will give the maximum speed of working. 
While intended primarily for direct current testing, the new instrument will be of assistance in testing alternating current voltmeters. This is done by the use of a transfer instrument which is equally correct on direct and alternating current; the transfer instrument, the potentiometer, and the voltmeter to be tested being connected in parallel with each other, first on alternating, then on direct emf. The change from alternating to direct voltage is accomplished by a quick-acting snap switch, the circuit being broken for so short a time that the deflection of the transfer instrument is maintained. This results in a saving of time and the avoidance of zero errors, and is a very accurate and convenient method.

Tests of this potentiometer in comparison with a five-dial standard potentiometer showed very small errors; the result (using the I25 volt range) being correct to within .or to .02 volt when the deflection of the galvanometer is small, and within .04 volt at the points of greatest error. For the higher ranges, and for the 5-volt range, the relative errors are equally small. This result will be improved by making a more accurate scale for the galvanometer, as some of the lines of the present scale are out of place by 0.2 scale division.

\section{SOURCES OF ERROR.}

The error in a meastirement with the deflection potentiometer is the algebraic sum of two distinct errors. The first is due to the error of adjustment of the coils constituting the potentiometer, considered as a null instrument, and affects the result when the galvanometer deflection is zero, as well as for any value of the deflection. This error is present in standard potentiometers of every form; but the accuracy possible in the adjustment of resistance coils is such that it can be made negligibly small in comparison with the second, namely, the error in the value of $\Delta E$, as given by the deflection of the galvanometer. The coils in the $\Sigma r$ circuit may not be correctly adjusted; the fine rheostat in the battery circuit cannot be perfectly compensated for all settings on the main dial; the scale of the galvanometer may be imperfectly graduated, and changes of temperature may affect the reading of the galvanometer. It is therefore necessary to investigate these points, in order to keep these errors small and be able to allow for them when necessary. Errors in the adjustment of the coils may be determined at any time by careful 
resistance measurements. If the error in the relative values of the coils in the main dial and of the volt box is such that corrections for the potentiometer as a null instrument are appreciable, a table of corrections can be made for the settings on the main dial. These corrections will then apply, whether the galvanometer is deflected or not. The accuracy required in the adjustment of the coils in the galvanometer circuit is much less than for the main dial and volt box coils, and errors due to this cause should be inappreciable, with good workmanship.

It is necessary to investigate the error in the deflection reading, due to the fact that the fine rheostat in plan 5 makes the resultant resistance of $r_{3}$ and $r_{6}$ no longer a constant. This error will be greatest when the main dial setting is a maximum and the coarse rheostat $r_{3}$ a minimum. To reduce the error we may let one-half the total resistance in the fine rheostat take the place of an equal amount in the coarse rheostat $r_{3}$; that is, one-half the fine rheostat may be considered part of the $r_{3}$ for which the values of $r_{6}$ are determined. Thus the fine rheostat will affect the resultant resistance one-half as much as would otherwise be the case. We have, then, the greatest error, in the present instrument, when $r_{3}=297.5$ and $r_{6}=6,8 \mathrm{I} 4$. In the middle position of the fine rheostat $5.5 \mathrm{ohms}$ is added to $r_{3}$, and the (normal) resultant resistance is

$$
\frac{303 \times 68 \mathrm{I} 4}{303+68 \mathrm{I} 4}=290 . \mathrm{I} \text { ohms. }
$$

Now let the remaining half of the fine rheostat be put in circuit, increasing $r_{3}$ to $308.5 \mathrm{ohms}$. The resultant resistance becomes

$$
\frac{308.5 \times 68 \mathrm{I} 4}{308.5+68 \mathrm{I} 4}=295 . \mathrm{I} 4 \mathrm{ohms} .
$$

For maximum setting of the dial, for the above values of $r_{3}$, the resistance in the galvanometer circuit between the contact point on the potentiometer wire and the zero end of the dial will be

$$
\begin{aligned}
& \frac{(290 . \mathrm{I}+9.9) 2500}{290 . \mathrm{I}+9 \cdot 9+2500}=267.86 \mathrm{ohms}, \\
& \frac{(295 \cdot \mathrm{I} 4+9.9) 2500}{295 \cdot \mathrm{I} 4+9.9+2500}=27 \mathrm{I} .87 \mathrm{ohms},
\end{aligned}
$$


the difference, 4 ohms, entering the total of 2,000 ohms in the $\Sigma r$ circuit, and requiring a compensation of 4 ohms. For the maximum value of $r_{3}$ the compensation required is $3.45 \mathrm{ohms}$, to be added or subtracted for the two extreme positions of the fine rheostat. The mean of these was taken, and the compensating rheostat made $2 \times 3.75$ ohms. The error for this extreme position of the fine rheostat, if not compensated, will be $\frac{4}{2000}$ of the deflection, or .06 scale division, which is about the limit of setting on a line. If this were the only source of error to be considered, it might be neglected; but it is desirable to avoid the accumulation of small errors, and by proper arrangement of circuits it is easy to arrange for compensation. It is evident that if we compensate for the error which enters at the maximum setting of the main dial, we introduce the full error at the zero position of the main dial, and a proportionally decreasing error as the dial setting increases to a maximum. This is not objectionable, however, for on low readings of an instrument under test the accuracy is necessarily low, and the greatest accuracy is desired at or near full scale deflection.

The method of combining the fine rheostat and the compensating rheostat in one simple dial may be seen diagrammatically in Fig. 5. It will be seen that the contact resistance of the lower end of the slider does not enter into the resistance of the wire $A B$, and the contact resistance of the upper end is in the galvanometer circuit. It is possible to put the fine rheostat in other places, but it is believed that this plan is the simplest and most satisfactory.

Errors due to imperfect graduation of the galvanometer scale depend upon the care taken by the maker, and may be determined by testing the galvanometer as an ammeter. The corrections to the deflection reading may be found for the galvanometer with its circuit by the following plan. The storage cell is removed, and the terminals to which it had been connected are joined by a wire of negligible resistance. This reduces to zero the first term in the numerator of equation ( $\mathrm{r} 7$ ), and the deflection is now proportional to $E$; in other words, this makes $E$ and $\Delta E$ identical. The value of $E$ may now be adjusted to give suitable values of the deflection, $E$ being measured by a standard potentiometer or calibrated voltmeter of suitable range. This method is necessary in plans 2, 3, and 4, 
where a zero setting on the main dial is impossible. For plans I and 5 the storage cell may be in position and the main dial set at zero.

Inasmuch as the 2,000 ohms of $\Sigma r$ contains, in the present design, 576 ohms of copper in the galvanometer, the deflection part of the reading will vary with the temperature. The galvanometer was found by trial to have practically zero temperature coefficient when used as an ammeter, the opposite coefficients of magnet and springs being sensibly equal. Hence the temperature coefficient of the galvanometer in use in the potentiometer is numerically that of the resistance of the $\Sigma r$ circuit, or $\frac{576}{2000} \times .003^{8}=.001$ I per degree C. Hence, for $10^{\circ} \mathrm{C}$ above or below that at which the instrument is adjusted, the error will be I.I per cent of the deflection, and for maximum deflection of 2.5 volts the error would be 0.03 volt. The simplest way to care for this is to have a table giving values of $\Delta E$ corresponding to various readings, at different temperatures. Another plan would be to have a resistance dial marked in degrees, so that $\Sigma r$ might be maintained at the correct value, for any temperature. A third plan would be to use the device known as the Bristol compensator, in which the rise and fall of mercury in a tube varies the length of a platinum wire in circuit. This could be made of such proportions as to just compensate for the effect of the variations in the galvanometer resistance. It is probable, however, that the first plan above is the best as well as the simplest, if all things are considered. For when the most accurate work is done, the temperature is usually kept near the standard, and as the galvanometer scale is not perfect, corrections will be required for the imperfections, if not for temperature. Hence if corrections are to be made at all, they may as well include those for temperature. For most purposes, even in the laboratory, the correction for temperature can be neglected.

It is important to note that in many applications of the deflection potentiometer the error of the result is almost independent of the error in the deflection part of the reading. For example, in testing a voltmeter the points checked are almost always a multiple of 5 or Io, so that the galvanometer reading is quite small, unless the voltmeter is considerably in error. The reading of the galvanometer 
is the correction to the voltmeter at the given point, and may be so read off, using the + and - signs marked on the galvanometer scale. Thus the error in the galvanometer deflection is a fraction of a per cent of the small correction to the voltmeter, and is negligible. In using a deflection potentiometer to determine a definite voltage, as IOO or I 20 volts, in order to mark the blank scale of an instrument, the method is a null one, and the result is independent of errors in the deflection part of the apparatus.

A point that should be investigated, in a proposed design, is the accuracy with which the current from the storage battery can be adjusted, in terms of the standard cell. While this depends partly upon the available number of steps in the fine rheostat, it also depends upon the galvanometer sensibility and the value of the total resistance in the circuit containing the standard cell and the galvanometer. In the present design the drop for the standard cell is taken from 5 Io ohms, which is shunted by 2,290 ohms, giving a resultant resistance of $4 \mathrm{I} 7$ ohms. To this must be added 576 ohms galvanometer resistance and 160 ohms internal resistance of the Weston standard cell, giving a total of I, I 53 ohms. If the current through the potentiometer wire is to be standardized in terms of the standard cell, to within one part in Io,0oo, then this fraction of the emf. of the cell, or .ooor volt, must give a noticeable deflection of the galvanometer. This emf. will send .OOOI $\div$ I,I53, approximately $\mathrm{I} \times \mathrm{IO}^{-7}$ ampere through this resistance, and the resulting deflection will be one-twentieth of a scale division, which is about the limit, but can be observed when the needle is over, or close to, a line. If greater accuracy of the standard current were desired, using this galvanometer, it could be secured by using two or more standard cells in series, and taking the drop from a greater portion of the potentiometer wire. This would double or treble the value of the small unbalanced emf. corresponding to a given error in the current, without increasing the resistance in the same proportion. An accuracy of one in I0,000 is, however, sufficient for all purposes for which this form of instrument would be used.

It has been asked whether an error would arise if there is considerable resistance in the source of the unknown emf. to be measured. This question arises because the denominator of the expression for the galvanometer current-equations (6) and ( I 7) - contains the term 
$R \frac{p-\mathrm{I}}{p^{2}}$, the resultant resistance of $\frac{R}{p}$ paralleled by the remainder of $R$. To test this point we may assume a resistance $r_{9}$ in the source of $E$ (page 23I of former article), when equations (I), (2), (3), and (4) remain unchanged, and (5) becomes

$$
i_{5}\left(R \frac{p-\mathrm{I}}{p}+r_{9}\right)+i_{4} \frac{R}{p}=E+i_{5} r_{9}
$$

which reduces to the form previously given, namely:

$$
i_{5} R \frac{p-I}{p}+i_{4} \frac{R}{p}=E
$$

In this case, as before, $E$ is taken to mean the potential difference at the terminals of the resistance $R$, which is the value it is desired to measure. The value of $i_{g}$ is thus unchanged, and the accuracy of the reading is independent of whether there is or is not resistance in the source of the pressure to be measured. The addition of resistance in the source would (theoretically) slightly affect the damping of the galvanometer, but in a practical case this effect would be inappreciable.

It is of interest to determine the magnitude of the error arising from the use of an external multiplier to extend the range. This error is, of course, zero when no current flows through the galvanometer, but is present when such a current flows. In equation (5) let $E$ increase to $E^{\prime}$, and let $R$ be increased by the addition of an external multiplier $R^{\prime}$. Then the equation becomes

$$
i_{5} R \frac{p-\mathrm{I}}{p}+i_{4} \frac{R}{p}+i_{5} R^{\prime}=E^{\prime}=E+i_{5} R^{\prime}
$$

which reduces to the original form of equation (5).

Considering the currents at the junction of $\frac{R}{p}$ and $R \frac{p-\mathrm{I}}{p}$ we have

$$
i_{4}-i_{5}-i_{g}=0
$$

From equations (2) and (5) we get

$$
\begin{aligned}
i_{5} & =\frac{E}{R}-\frac{\mathrm{I}}{{ }^{2}} i_{g} \\
& =\frac{E}{R}-\frac{\mathrm{I}}{p} \cdot \frac{\Delta e}{\sum r}
\end{aligned}
$$


Further,

$$
\begin{aligned}
E^{\prime} & =E+i_{5} R^{\prime} \\
& =E+R^{\prime}\left(\frac{E}{R}-\frac{\mathrm{I}}{p} \cdot \frac{\Delta e}{\Sigma r}\right) \\
& =E\left(\mathrm{I}+\frac{R^{\prime}}{R}-\frac{R^{\prime}}{p \Sigma r} \cdot \frac{\Delta e}{E}\right)
\end{aligned}
$$

When no current flows through the galvanometer this reduces to the ordinary formula for the use of an external multiplier,

$$
E^{\prime}=E\left(I+\frac{R^{\prime}}{R}\right)
$$

Hence, when the galvanometer current is not equal to zero, and the ordinary factor $\mathrm{I}+\frac{R^{\prime}}{R}$ is applied to the reading of the instrument to determine the emf. to be measured, the relative error $\epsilon$ is given by the formula

$$
\begin{aligned}
\epsilon & =\frac{\frac{R^{\prime}}{p \sum r} \cdot \frac{\Delta e}{E}}{\frac{R+R^{\prime}}{R}} \\
& =\frac{R R^{\prime}}{\left(\sum r\right)\left(R+R^{\prime}\right)} \cdot \frac{\Delta e}{p E}
\end{aligned}
$$

It is desirable to express the small quantity $\Delta e$ (which is to be distinguished from $\Delta E$ ) in terms of $E$, as follows:

$$
\begin{aligned}
\Delta e & =\frac{r_{1}}{r_{1}+r_{2}+r_{3}} e_{1}-\frac{E}{p} \\
& =\frac{p s-E}{p} \\
& =\frac{\Delta E}{p}
\end{aligned}
$$

Equation (28) thus becomes

$$
\epsilon=\frac{R R^{\prime}}{(\Sigma r)\left(R+R^{\prime}\right) p^{2}} \frac{\Delta E}{E}
$$


The reason for this correction term is simply that when the resistance $\frac{R}{p}$ is shunted by a larger value than the normal amount, $R \frac{p-\mathrm{I}}{p}$, namely, by $R \frac{p-\mathrm{I}}{p}+R^{\prime}$, the resultant resistance is increased, and if the galvanometer gave a correct indication before, it will now give too small a deflection. This can be taken care of by applying the correction formula (29), or we may arrive at the same result by calculating the increment to $\Sigma r$, and increasing $\Delta E$ in the same ratio. Thus, if $R$ is increased by an amount $R^{\prime}$

$$
\Delta(\Sigma r)=\frac{\frac{R}{p}\left(R \frac{p-\mathrm{I}}{p}+R^{\prime}\right)}{R+R^{\prime}}-R \frac{p-\mathrm{I}}{p^{2}}
$$

which reduces to the form

$$
\Delta(\Sigma r)=\frac{R R^{\prime}}{\left(R+R^{\prime}\right) p^{2}}
$$

Hence the relative error in $\Sigma r$ is

$$
\frac{\Delta(\Sigma r)}{\Sigma r}=\frac{R R^{\prime}}{(\Sigma r)\left(R+R^{\prime}\right) p^{2}}
$$

and the relative error in the observed value of the unknown emf. is

$$
\begin{aligned}
\epsilon & =\frac{\Delta \Sigma r}{\Sigma r} \cdot \frac{\Delta E}{E} \\
& =\frac{R R^{\prime}}{(\Sigma r)\left(R+R^{\prime}\right) p^{2}} \frac{\Delta E}{E}
\end{aligned}
$$

In equation (26), if a positive value of $\Delta e$ corresponds to an excess of the potentiometer setting over $\frac{E}{p}$, or to values of $E$ lower than that for which the galvanometer current is zero, $E^{\prime}$ will be lower than $E\left(\mathrm{I}+\frac{R^{\prime}}{R}\right)$. When $E$ is higher than the value for which the galvanometer current is zero, the correction term in (26) becomes positive, and $E^{\prime}$ is greater than $E\left(\mathrm{I}+\frac{R^{\prime}}{R}\right)$. 


\section{OUTLINE OF METHOD OF DESIGN.}

In designing a deflection potentiometer, the central and determining feature is the galvanometer. From the current $I$ for one scale division, the number of divisions $m$ it is to give per volt of $\Delta E$, and the total resistance for critical damping, which latter is substituted as $\Sigma r$, we may determine an approximate value of $p$ from equation (II), namely:

$$
\Sigma r=\frac{\mathrm{I}}{p m I} \text { or } p=\frac{\mathrm{I}}{(\Sigma r) m I}
$$

As the resistance for critical damping is not sharply defined, we may modify the result somewhat, to give $p$ a convenient integral value. From this tentative value of $p$ and the maximum emf. which it is desired to measure, we may find the fall of potential on $A B$, which is $\frac{E}{p}$. For plan 5 this should be approximately a multiple of I.7 volts, and will show how many storage cells will be required. Having fixed the value of $p$, determine $\Sigma r$ by substitution in equation (I I). Deduct 5 or Io per cent from $\Sigma r$ for a calibrating coil, and from the remainder deduct the resistance of the galvanometer. The rest of $\Sigma r$ is to be divided between $R \frac{p-I}{p^{2}}$ and the resistance through the potentiometer proper, including the compensating resistance $r_{4}$. This latter makes the resistance through the potentioneter (from the point of contact on the wire $A B$ to the point $A$ ) constant and equal to one-fourth of the total equivalent resistance of the circuit supplied by the storage cells. As the emf. of these cells, when freshly charged, will be about 2.I volts each as a maximum, this total equivalent resistance will be approximately $\frac{2 . \mathrm{I}}{\mathrm{I} \cdot 7} r_{w}$, where $r_{w}$ is the resistance of the wire $A B$; hence the constant resistance through the potentiometer will be approximately $\frac{2 . \mathrm{I}}{6.8} r_{w}$. As a first approximation, divide the rest of $\Sigma r$ equally between this and $R \frac{p-I}{p^{2}}$, and determine the values of $r_{w}$ and $R$. It is desirable, for adjusting and checking, that each step of $r_{w}$ have an even and usual value of 
resistance, as 20,50 , or Ioo olnms; with this in view, $r_{w}$ may be chosen and $R$ inodified to suit. $R$ may then be given an even value, the difference being taken up in the calibrating coil. It is desirable to make $R$ large, to reduce energy loss and consequent heating of the volt box coils; it is also desirable to make $r_{w}$ large, as the drain on the storage cells is thereby reduced, and slight variations in rheostat contacts do not then exert as much influence on the current strength.

The number of steps of the nain dial is determined by two considerations. The smaller each step, the more accurate the instrument, because less of the total quantity is read by deflection. To go too far in this direction increases the size and cost of the instrument for a given range, and reduces the convenience and speed of working. The minimum number of steps is determined by the range of the galvanometer, assuming that emfs. are to be measured continuously from the lowest to the highest.

The mechanical design should conform to good practice in such matters as reliable dial contacts, subdivision of coils in the volt box to reduce the dielectric stress per coil, and sufficient area in these coils to keep the heating small. The guard box around these coils is an essential feature, particularly when the instrument is to be used in damp weather. The grouping of the parts of the apparatus should be made with a view to rapidity and convenience of working, and will depend upon the judgment of the designer.

\section{CONCLUSION.}

In this and in the preceding article stress has been laid upon the desirability of using a pivoted portable form of galvanometer. This has been done because the aim has been to produce instruments that can be quickly and conveniently operated and moved from one place to another without requiring the special setting up which is necessary with reflecting galvanometers having delicate suspensions and small clearance between coil and pole pieces. Any galvanometer can be used which has a good zero and a constant that is reasonably permanent. It is desirable, if a reflecting galvanometer is to be used, to employ a taut suspension above and below, heavy enough to give quick working and good zero. For a given set of conditions the sensibility required can be determined, and the suspensions can 
then be chosen to give this sensibility. The matter of critical damping should be taken into account, as before mentioned.

In the case of a deflection potentiometer for current measurements in connection with suitable shunts, which might have say I5O millivolts as the upper limit of its range, the small amount of power available imposes much greater requirements upon the galvanometer than in the case of an instrument for the measurement of voltage, such as the one above described. The design of such a potentiometer for current measurements has been begun.

While the potentiometer for voltage measurements, as described in this article, is best adapted to laboratory use, where voltmeters of various ranges must be tested, a simpler and less expensive instrument would answer all requirements in central station work. For example, a range of IO5 to I 25 volts, with provision to double and quintuple the range, would cover the most important requirements of the light and power station. The sensibility in this case would not need to be as great as for the laboratory instrument, so that a less sensitive galvanometer would be satisfactory. Preliminary work on the design of such an instrument has been begun.

WASHINGTON, October II, I907. 